la revue La revue pour l'histoire du CNRS

PoUR L LHISTORE Du CNRS $\quad 5 \mid 2001$

Des laboratoires à l'étranger

\title{
Les sciences et leurs langages
}

Textes réunis et publiés par Henri Vérine. Éditions du comité des

travaux historiques et scientifiques, Paris, 2000

Laurent Pinon

\section{OpenEdition}

Journals

Édition électronique

URL : https://journals.openedition.org/histoire-cnrs/395

DOI : $10.4000 /$ histoire-cnrs.395

ISSN : 1955-2408

Éditeur

CNRS Éditions

Édition imprimée

Date de publication : 5 novembre 2001

ISBN : 978-2-271-05925-3

ISSN : 1298-9800

\section{Référence électronique}

Laurent Pinon, "Les sciences et leurs langages », La revue pour l'histoire du CNRS [En ligne], 5 | 2001, mis en ligne le 06 mars 2006, consulté le 20 mai 2021. URL : http://journals.openedition.org/histoirecnrs/395 ; DOI : https://doi.org/10.4000/histoire-cnrs.395

Ce document a été généré automatiquement le 20 mai 2021.

Comité pour l'histoire du CNRS 


\section{Les sciences et leurs langages}

Textes réunis et publiés par Henri Vérine. Éditions du comité des

travaux historiques et scientifiques, Paris, 2000

\section{Laurent Pinon}

1 Sous le titre Les sciences et leurs langages, ce volume réunit les actes de deux colloques de la section des sciences du Comité des travaux historiques et scientifiques (CTHS), tenus à Amiens en 1994 et à Aix-en-Provence en 1995. Il séduit d'emblée tant par la qualité matérielle de sa composition que par la cohérence des sujets traités, qui couvrent un large spectre des domaines scientifiques. Les trois parties de l'ouvrage traitent en effet des "sciences de la matière et mathématiques", des "sciences de la vie » et des «sciences humaines». Chacune des vingt-huit contributions est accompagnée d'un résumé et d'une bibliographie qui facilitent l'orientation du lecteur.

Ces évidentes qualités formelles, ainsi que la grande richesse de certaines contributions, ne peuvent qu'inciter à regretter la grande hétérogénéité de qualité des textes et, surtout, l'absence d'une problématique générale ou d'une mise en perspective d'ensemble qui aurait notablement accru la portée du volume. à cet égard, le texte qui fait office d'introduction tient presque de la caricature : totalement décousu, il offre une fastidieuse liste de catégories de métaphores, d'étonnantes "variations conceptuelles autour du un et du zéro » ou encore une douteuse comparaison de la notion d'imaginaire en mathématiques et en psychologie.

3 D'autres contributions suscitent la même perplexité, comme une étude sur la " création nomenclaturale » en botanique et dans quelques sciences apparentées comme la phytosociologie, l'ethnophytosociologie ou la symphytosociologie (?) qui livre une fastidieuse énumération de mécanismes nomenclaturaux tout en mélangeant la nomenclature proprement dite avec de simples qualificatifs plus ou moins étroitement liés aux plantes. L'étonnant article sur la taxinomie des Bryozoaires eurystomes a au moins le mérite de montrer comment une petite poignée de spécialistes à travers le monde nomment les espèces nouvelles, mobilisant avec imagination références confidentielles ou jeux de mots incompréhensibles. Tout cela pour conclure de manière stupéfiante que l'« éventail des catégories dans lesquelles entrent les différents taxons décrits par les bryozoologues est le reflet de la diversité humaine »! 
On trouve heureusement de remarquables contributions dans lesquelles la précision de l'information s'accompagne d'analyses fines et fécondes. C'est le cas par exemple de celles de Renée et Christian Bange sur le vocabulaire de l'endocrinologie et de la physiologie, de celle de Suzanne Débarbat sur la nomenclature du système métrique, ou des deux études de Simone Dumont et de Monique Gros sur les appellations des corps célestes qui montrent comment et avec quelles difficultés des standards internationaux se mettent en place. Le problème des traductions est largement traité, notamment à travers le très bon article d'Andrée Le Bœuffle sur le vocabulaire astronomique de Cicéron.

5 De manière générale, les présentations restent trop souvent descriptives, là où une analyse des rapports de force, internationaux ou sociaux, qui s'exercent à travers les nomenclatures aurait probablement pu apporter d'autres éléments de compréhension. Il n'en reste pas moins que, sur des questions aussi importantes que la persistance des noms anciens, les lacunes des langues vernaculaires, le rôle des éponymes, ou la transition entre appellations locales et standards internationaux, les exemples concrets et originaux, souvent développés avec pédagogie, offrent véritablement matière à penser.

\section{AUTEUR}

\section{LAURENT PINON}

École normale supérieure, département d'histoire 